Open Access

\title{
A review of Karl Maton, Susan Hood \& Suellen Shay (eds.), Knowledge Building: Educational Studies in Legitimation Code Theory, Abingdon and New York: Routledge, 2016. xvii + 264 pp., US\$160.00 (hbk)
}

Yan Liu', ${ }^{1,2}$ and Hui Yu ${ }^{1 *}$ (D)

\footnotetext{
* Correspondence: yuh@bnu.edu.cn ${ }^{1}$ School of Foreign Languages and Literature, Beijing Normal University, Beijing 100875, People's Republic of China

Full list of author information is available at the end of the article
}

\begin{abstract}
As educational linguistic researchers, we seek to explore the relations between language, society, knowledge and education. This current volume appeals to us since its efforts coincide with our research interest. It addresses two weaknesses commonly found in research into education and society: knowledge blindness and segmentalism. Part I demonstrates reflexively how LCT as an explanatory framework could be applied to overcome segmentalism. Part II applies LCT to six studies through uncovering the underlying principles of knowledge practice. Part III includes an architectural glossary. We conclude by discussing the strengths and insufficiencies of the volume. Despite the insufficiencies, this volume will prove to be a lasting inspiration for readers of different fields.
\end{abstract}

Keywords: Knowledge building, Knowledge practice, Legitimation code theory, Systemic functional linguistics, Specialization, Semantics

As educational linguistic researchers, we seek to explore the relations between language, society, knowledge and education. Such inquiries coincide with the research domain of educational sociologists. Inspired by the fruitful dialogue between systemic functional linguistics (SFL) and Bernsteinian sociology, we found it helpful to draw from Maton, who inherited and developed Bernstein's conceptualization of knowledge.

The current volume addresses two weaknesses commonly found in research into education and society: knowledge blindness and segmentalism. Knowledge blindness refers to the failure to regard knowledge itself as an object of serious study, while segmentalism hinders dialogues between different methodologies, theories, disciplines and substantive studies. Legitimation Code Theory (LCT) was introduced by Maton (2014) to overcome these difficulties. If Maton (2014) was theory-oriented, the present volume is application-oriented. It aims at uncovering the underlying principles of various forms of knowledge practices, illustrating how LCT could help account for knowledge building within and beyond education.

(c) The Author(s). 2018 Open Access This article is distributed under the terms of the Creative Commons Attribution 4.0 International License (http://creativecommons.org/licenses/by/4.0/), which permits unrestricted use, distribution, and reproduction in any medium, provided you give appropriate credit to the original author(s) and the source, provide a link to the Creative Commons license, and indicate if changes were made. 
This volume consists of 12 chapters. Chapter 1 introduces the framework and summarizes the key concepts. Chapters 2-5 (Part I) demonstrate reflexively how LCT as an explanatory framework could be applied to overcome segmentalism. Chapters 6-11 (Part II) apply LCT to six studies across different disciplines. Part III (Chapter 12) includes an architectural glossary describing how concepts interrelate within the framework.

Specifically, Chapter 1 elaborates specialization dimension and semantics dimension of LCT. Specialization conceptualizes knowledge-knower structures of knowledge practices in terms of epistemic relation and social relation; semantics conceptualizes semantic structures in terms of semantic gravity and semantic density. Part I (Chapters 2-5) is foregrounded by LCT's position between meta-theories and substantive research studies. This mediating position privileges it to function as a bridge transcending the divide between theory and data (Chapter 2), between qualitative and quantitative methodologies (Chapter 3), between theory and practice (Chapter 4), and between disciplines in research (Chapter 5). Those bridging processes have been explicated in the following case studies: the effects of online constructivist pedagogy on Chinese overseas students in Australia through the creation of a translation device between theory and data (Chapter 2); the pedagogic efficiency of different subjects brought by integration of information and technology through a quantitative questionnaire with LCT concepts (Chapter 3); the creation of e-learning environment in a museum with LCT concepts embedded in the design (Chapter 4); an interdisciplinary project on knowledge building in secondary school history and biology classrooms in terms of semantics from LCT and power trio from SFL (Chapter 5).

Part II is intended to be the application of the theory in Part I. Chapter 6 explores how storytelling as a form of knowledge transmission evolves from everyday practice into academic practice. A combined approach of genre analysis from SFL and specialization and semantics dimensions of LCT reveals the differences of storytelling in social sciences and humanities. Chapter 7 constructs a conceptual framework analyzing knowledge progression in vocational curricula. In this study, knowledge is not only translated into curriculum design, but also translated for the purpose of solving practical problems. Chapter 8 argues Secondary School English Literacy Studies is a knower-code knowledge practice. Examination questions and three successful compositions at different ages are analyzed in order to explore how to master a cultivated gaze. Chapter 9 makes use of semantics dimension of LCT to analyze students' responses to one physics question and the basis of their achievements. Results suggest that there exists an appropriate semantic range for students' success. Chapter 10 employs specialization dimension of LCT and investigates how music students position outstanding musicians and themselves through the academic papers by them. Chapter 11 studies the nature of masonic planks and how it serves as a tacit pedagogy. By enacting concepts of semantic gravity and semantic density from LCT and concepts from SFL, the researcher discovers that all the masonic planks are characterized by a weaker semantic gravity and a stronger semantic density.

The current volume gives a powerful demonstration of the strengths of LCT. First, the application of LCT stimulates the interdisciplinary interaction between education, sociology and linguistics. Nearly all the studies in Part II adopt a combined approach of LCT and SFL. While SFL (e.g. genre analysis in Chapter 6) helps uncover the 
legitimation codes of knowledge practices, LCT helps interpret how linguistic resources (e.g. grammatical metaphor and nominalization in Chapter 11) are reflected in knowledge practice. These combined efforts contribute to uncovering knowledge building in education and society. Second, LCT's strong adaptability and feasibility is reflected in a wide range of studies. Maton argues convincingly that "all practices are construed as languages of legitimation" (p.10). Apart from educational knowledge practices, the theory can also be applied to other areas including the study of storytelling in Chapter 6 and masonic planks in Chapter 11.

However, there still exist some insufficiencies with the current volume. First, all the studies center around specialization and semantics dimension, excluding the other three dimensions of LCT (autonomy, density and temporality). The latter three dimensions are hardly explained in terms of its applicability. Second, this volume is heavily loaded with educational sociological concepts (e.g. ontology, epistemology) that may cause reading difficulties for researchers outside the area. A provision of a glossary on these technical terms would have helped. Thirdly, most of the research in Part II is by nature qualitative using data such as interviews, students' responses to a question, student's writing, national documentary, thus failing to be quantitative as Chapter 5 proclaims.

Despite the insufficiencies, this volume proves to be an inspiration for readers of different fields. First, it helps to bridge the gaps haunting humanities and social sciences. Second, it is a good example of how interdisciplinary research can be done by enacting complimentary theories for explanatory purposes, which is, in this case, the combination of SFL and LCT. Thirdly, it offers educational theorist advice concerning policy making and curriculum design. Fourthly, it provides teachers with implications on how to promote knowledge building in their teaching practice.

Funding

This study is a part of the project "A Study on Knowledge Structure of Educational Discourse from the Perspective of Genre Typology" supported by the Ministry of Education of China (project number 16YJA740044).

Authors' contributions

Both authors read and approved the final manuscript.

Competing interests

The authors declare that they have no competing interests.

\section{Publisher's Note}

Springer Nature remains neutral with regard to jurisdictional claims in published maps and institutional affiliations.

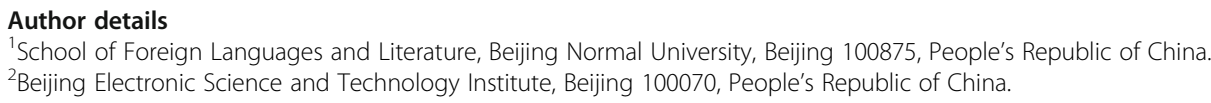

